\begin{tabular}{l|l|l|l|l} 
Revue suisse Zool. & Tome 81 & Fasc. 4 & p. 839-844 & Genève, décembre 1974
\end{tabular}

\title{
Détermination de l'âge de Mus musculus Linné par l'usure de la dentition
}

par

\section{A. KELLER}

Avec 9 figures

\section{INTRODUCTION}

Le but de cette étude est d'apporter une contribution à la connaissance de la souris Mus musculus L. En effet, je pense que la détermination de l'âge, par l'usure de la dentition, peut répondre à certaines exigences dans une étude de populations. Je trouve dans la littérature plusieurs études semblables concernant des genres voisins de Muridés, en particulier sur Apodemus Kaup.. Par contre, un seul auteur, semble-t-il a traîté Mus musculus, soit BrEAKEY (1963). Ses résultats ne s'appliquent cependant pas d'une façon pratique aux populations de Mus de nos régions (voir discussion).

\section{MATÉRIEL ET MÉTHODE}

Cette étude est basée sur le fait bien connu de l'usure progressive des dents en rapport avec l'âge. J'ai réparti les spécimens d'élevage et ceux capturés en 5 classes d'âge (voir tab.).

Les spécimens d'élevage ont servi à l'établissement de l'âge dans les classes, de la manière suivante:

$$
\begin{aligned}
& \text { Classe 1: individus de } 3 \text { semaines } \\
& \text { » 2: " \ } 1 \text { à } 2 \text { mois } \\
& \text { »3: ॥ } \quad 3 \text { à } 5 \text { mois } \\
& \text { » 4: \ \6 } 6 \text { à } 10 \text { mois } \\
& \text { »5: » } \gg 11 \text { mois et plus }
\end{aligned}
$$


La différence de temps écoulé entre les 3 premières classes et les 2 dernières traduit l'usure moindre des dents lorsque l'animal vieilli.

\begin{tabular}{|c|c|c|c|}
\hline \multirow{2}{*}{ CLASSES } & \multicolumn{2}{|c|}{ nombre de spécimens examinés } & \multirow{2}{*}{ TotAL } \\
\cline { 2 - 3 } & élevage & captures & \\
\hline 1 & 13 & 4 & 17 \\
\hline 2 & 22 & 60 & 82 \\
\hline 3 & 20 & 28 & 48 \\
\hline 4 & 13 & 16 & 29 \\
\hline 5 & 2 & 10 & 12 \\
\hline TotAL & 70 & 118 & 188 \\
\hline
\end{tabular}

Après cette répartition et élaboration des classes avec des spécimens d'élevage, j'ai appliqué ce système sur des individus capturés dans des habitations. Les quelques pièges que j'ai posés dans la campagne n'ont rien donné. Le matériel n'est pas très abondant comme le montre le tableau (188 spécimens), mais il me parait suffisant pour tirer des conclusions significatives.

$94,5 \%$ du matériel examiné, provient de la région genevoise, et le 5,5\% d'autres régions de Suisse. Ce matériel est déposé au Muséum d'Histoire naturelle de Genève.

Seules les molaires supérieures ont été prises en considération et les 9 figures représentent toujours le côté gauche.

\section{CLASSE I}

\section{RÉSULTAT}

Tous les spécimens dont $\mathrm{M}^{1}$ et $\mathrm{M}^{2}$ sont seules présentes, sont mis dans la $1^{\text {re }}$ classe (fig. 1).

\section{CLASSE 2}

$\mathrm{M}^{3}$ visible, usure de $\mathrm{M}^{1}$ comme dans la classe 1 . Jusqu'à l'âge de 4 semaines, les surfaces usées de t4-t5-t6 sont séparées par la cloison émaillée (fig. 2), alors qu'à 5 semaines, ces mêmes surfaces usées sont réunies en une seule zone d'abrasion (fig. 3-4). La zone usée de $\mathrm{t} 7$ est indépendante de celle de t8 à 4 semaines (fig. 2), mais ordinairement, dès les $6^{\mathrm{e}}, 7^{\mathrm{e}}$ et $8^{\mathrm{e}}$ semaines, ces deux zones d'usure sont 
réunies (fig. 3-4). Cependant, il peut y avoir quelques variations, car j'ai constaté chez certains spécimens âgés d'un mois que les surfaces usées de $t 7$ et t8 étaient déjà réunies en une seule zone d'abrasion.

\section{CLASSE 3}

Une seule cloison émaillée entre les deux surfaces d'usure de $\mathrm{t} 15$ et t16 (figs 5-6). Les $\mathrm{t} 1 \mathrm{à} \mathrm{t} 3$ sont réunis en une seule zone d'abrasion. Sur le côté interne de $\mathrm{M}^{1}$, la bordure émaillée qui sépare $\mathrm{t} 4 \mathrm{de} \mathrm{t} 7$ est double avant 4 mois (fig. 5) et simple
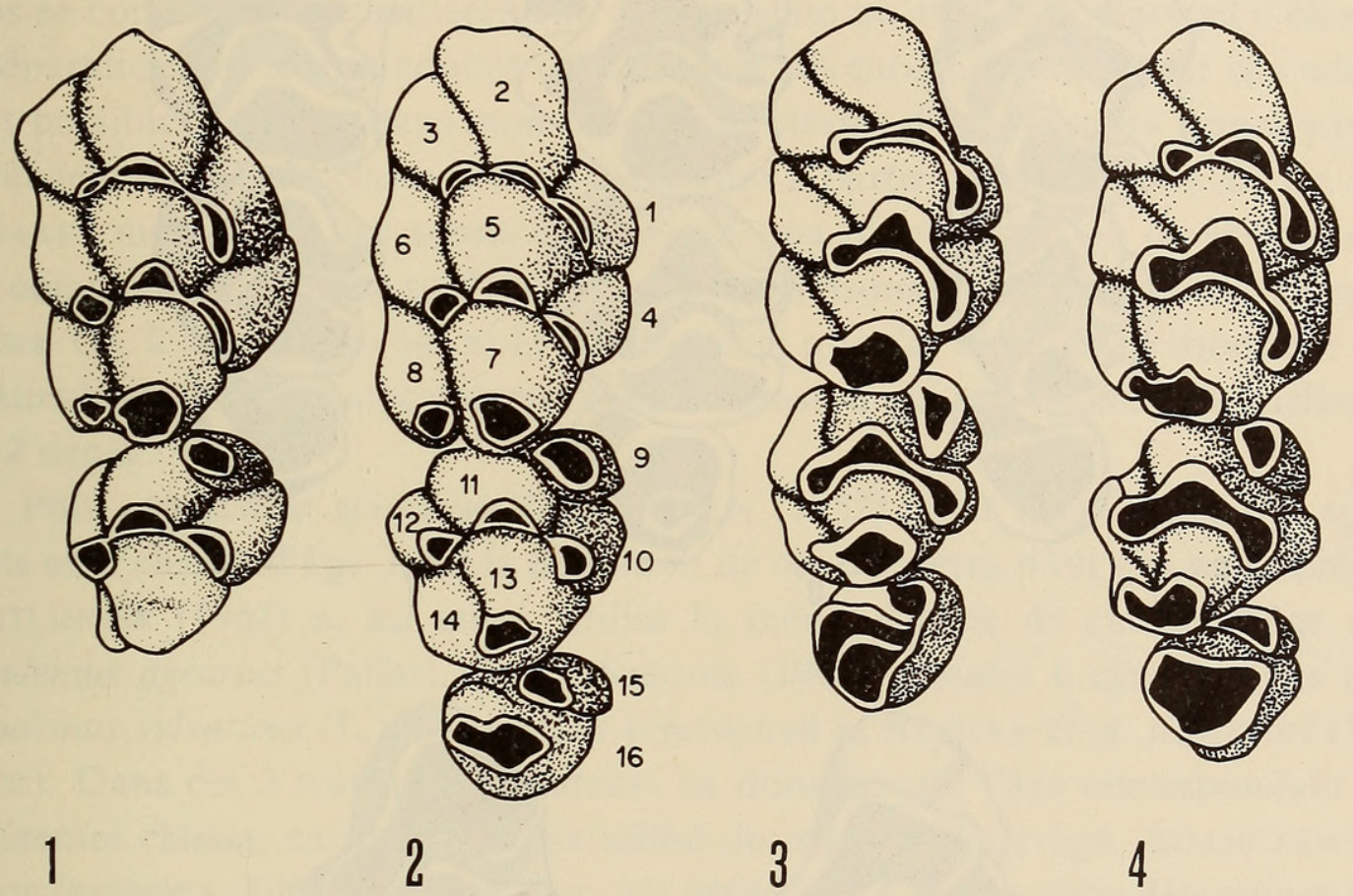

FIGs. 1-4.

1: classe 1,3 semaines d'âge; $2-4$ : classe 2,1 à 2 mois d'âge.

(fig. 2, numérotation des tubercules $(\mathrm{t})$ )

après 4 mois et demi (fig. 6). La largeur de la surface usée de $t 7$ et $t 8$ est plus d'une fois et demi plus grande que celle de $\mathrm{t} 7$ et $\mathrm{t} 8$ de la classe précédente (figs 4-5).

\section{CLASSE 4}

Il apparaît sur $\mathrm{M}^{1}$ une boucle émaillée supplémentaire qui se lie à t6 (figs 7-8). Vers 10 mois et demi, t4-t5-t6-t7 et t8 sont unis en une seule zone d'abrasion (fig. 8). Il en va souvent de même pour $\mathrm{t} 15$ et t 16 , qui montrent également une surface totalement usée. Les cloisons émaillées persistent quelquefois chez $\mathrm{M}^{3}$, et laissent apparaitre trois anneaux (fig. 8), que l'on trouve déjà à la fin de la classe 3 (fig. 6). 


\section{CLASSE 5}

Réunion complète des zones d'abrasion chez $\mathrm{M}^{2}$ ( $\mathrm{t}$ 9 àt 14$)$, ainsi que chez $\mathrm{M}^{3}$ (t15 et t16) (fig. 9).
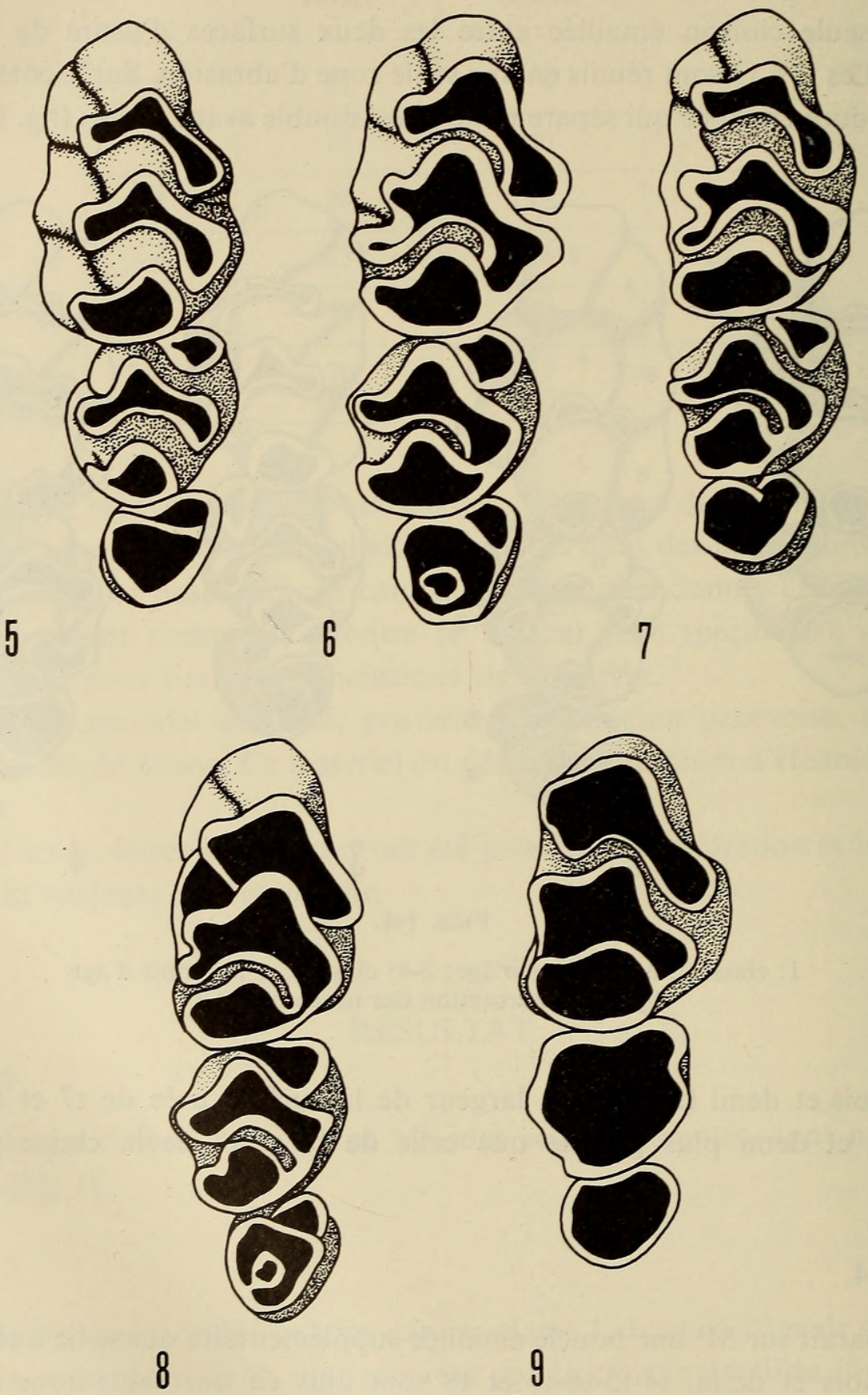

8

Figs. 5-9.

$5 \cdot($ : classe 3, 3 à 5 mois d'âge; 7-8: classe 4,6 à 10 mois d'âge; 9: classe 5,11 mois et plus. 


\section{DISCUSSION}

Dans son travail, Breakey (1963) divise les classes d'âge en 10 catégories de 2 mois chacune, ceci jusqu'à 18 mois. Toutefois, il fait une exception pour la $1^{\text {re }}$ classe qui ne dure qu'un mois. Le nombre de classes établies par BREAKEY est trop élevé, à mon avis, et amène une certaine confusion. Les chevauchements inévitables ne permettent pas une appréciation aisée de l'âge, au moins pour les populations de nos régions (le matériel de BreaKey provient de la baie de San Francisco).

Les classes que j'ai établies ici s'intercalent en gros entre celle de BreAKeY, mais ne concordent pas entièrement. J'ai constaté qu'avec mon matériel d'élevage, la séparation des jeunes adultes qui ne se sont pas encore reproduits et des adultes était possible. En effet, dans mon échelle des classes d'âge, tous les jeunes adultes se rangent dans la $2^{\mathrm{e}}$ classe et tous les adultes à partir de la $3^{\mathrm{e}}$ classe. La fig. 2 de BREAKEY montre sur une souris de 12 mois, une zone d'abrasion de $\mathrm{M}^{3}$ qui n'est pas complète sur toute sa surface, puisque des cloisons émaillées sont encore apparentes. Or, à cet âge, les $\mathrm{M}^{2}$ et $\mathrm{M}^{3}$ du matériel que j'ai examiné ne montrent plus aucune trace de cloison et ont une zone d'abrasion qui couvre toute la surface de ces 2 dents.

Pour Apodemus sylvaticus (L.), Felten (1952) avait déjà réparti ses spécimens en 5 classes d'âge, mais la définition de ces dernières n'est pas assez précise. HaitLINGER (1962) a, lui aussi, utilisé la même échelle de classes d'âge chez Apodemus agrarius (Pallas). Enfin, STEINER (1968) a établi 6 classes d'âge pour Apodemus sylvaticus (L.), A. microps Kratochvil et Rosicky et A. flavicollis (Melchior). Dans ces 3 travaux, les auteurs ne donnent pas l'âge correspondant aux différentes classes, car ils n'ont pas utilisé du matériel d'élevage. AdAMCZEwSKAANDRZEJEWSKA (1967), pour sa part, n'a défini que 4 classes pour déterminer l'âge chez Apodemus flavicollis; par contre, l'âge des mulots de chacune de ces 4 classes peut être déterminé, mais pas d'une façon aussi précise qu'avec l'utilisation de 5 classes.

\section{RÉSUMÉ}

L'auteur se base sur les différents stades d'usure des molaires supérieures gauches pour déterminer l'âge de Mus musculus. Il réparti ensuite ces différents stades dans 5 classes qu'il divise de la manière suivante: $1^{\text {re }}$ classe: jusqu'à 3 semaines. $2^{\mathrm{e}}$ classe: 1 à 2 mois. $3^{\mathrm{e}}$ classe: 3 à 5 mois. $4^{\mathrm{e}}$ classe: 6 à 10 mois. $5^{\mathrm{e}}$ classe: 11 mois et plus.

\section{ZUSAMMENFASSUNG}

Für die Altersbestimmung von Mus musculus verwendet der Autor die verschiedenen Stufen der Abnutzung der linken oberen Molaren. 5 Altersklassen 
werden errichtet: 1. Klasse: bis 3 Wochen; 2. Klasse: 1-2 Monate; 3. Klasse: 3-5 Monate; 4. Klasse: 6-10 Monate; 5. Klasse 11 Monate und mehr.

\section{REMERCIEMENTS}

Ce travail a été réalisé sous la direction du professeur V. Aellen, directeur du Muséum d'Histoire naturelle de Genève, que je remercie vivement ici pour ses conseils. Ma reconnaissance va également à MM. V. Mahnert et F. Baud pour leur précieuse aide.

\section{BIBLIOGRAPHIE}

Adamczewska-Andrzejewska, K. A. 1967. Age reference model for Apodemus flavicollis (Melchior). Ekol. Pol., ser. A, 15 (41): 787-790.

Breakey, D. R. 1963. The breeding season and age structure of feral house mouse populations near San Francisco Bay, California. J. Mammal. 44 (2): 153-158.

Felten, H. 1952. Untersuchungen zur Ökologie und Morphologie der Waldmaus (Apodemus sylvaticus L.) u. der Gelbhalsmaus (Apodemus flavicollis Melchior) im Rhein-Main-Gebiet. Bonn. zool. Beitr. 3-4: 187-206.

Haitlinger, R. 1962. Morphological variability in Apodemus agrarius (Pallas). Acta theriol. 6 (8): 239-257.

SteINER, H. M. 1968. Untersuchungen über die Variabilität und Bionomie der Gattung Apodemus (Muridae, Mammalia) der Donau-Auen von Stockerau (Niederösterreich). Z. wiss. Zool. 177 (1-2): 1-96.

Adresse de l'auteur :

Muséum d'Histoire naturelle

Case postale 284

CH-1211-Genève 6

Suisse 


\section{$2 \mathrm{BHL}$ Biodiversity Heritage Library}

Keller, A. 1974. "Détermination de l'âge de Mus musculus Linné par l'usure de la dentition." Revue suisse de zoologie 81, 839-844.

https://doi.org/10.5962/bhl.part.76046.

View This Item Online: https://www.biodiversitylibrary.org/item/126812

DOI: https://doi.org/10.5962/bhl.part.76046

Permalink: https://www.biodiversitylibrary.org/partpdf/76046

\section{Holding Institution}

Smithsonian Libraries

\section{Sponsored by}

Biodiversity Heritage Library

\section{Copyright \& Reuse}

Copyright Status: In Copyright. Digitized with the permission of the rights holder.

Rights Holder: Muséum d'histoire naturelle - Ville de Genève License: http://creativecommons.org/licenses/by-nc-sa/3.0/

Rights: https://www.biodiversitylibrary.org/permissions/

This document was created from content at the Biodiversity Heritage Library, the world's largest open access digital library for biodiversity literature and archives. Visit BHL at https://www.biodiversitylibrary.org. 